\title{
COVID-19 and Cytomegalovirus Co-infection: A Challenging Case of a Critically III Patient with Gastrointestinal Symptoms
}

\author{
Pedro HF Amaral ${ }^{1}$, Bernadete MC Ferreira² ${ }^{2}$, Sergio Roll ${ }^{1}$, Precil DMM Neves², Luca GA Pivetta $^{1}$, Sara Mohrbacher $^{2}$, Eduardo RM Dias $^{1}$, \\ Victor AH Sato², Érico S Oliveira², Leonardo VB Pereira², Alessandra M Bales², Luciana L Nardotto², Jéssica N Ferreira², \\ Américo L Cuvello-Neto ${ }^{2}$, Pedro R Chocair ${ }^{2}$ \\ ${ }^{1}$ Gastrointestinal Surgery Service, Oswaldo Cruz German Hospital, São Paulo-SP, Brazil \\ ${ }^{2}$ Internal Medicine Service, Oswaldo Cruz German Hospital, São Paulo-SP, Brazi
}

Received: $24 / 07 / 2020$

Accepted: $30 / 07 / 2020$

Published: 02/09/2020

\begin{abstract}
How to cite this article: Amaral PHF, Ferreira BMC, Roll S, Neves PDMM, Pivetta LGA, Mohrbacher S, Dias ERM, Sato VAS, Oliveri ES, Pereira LVB, Bales AM, Nardotto LLL, Ferreira JN, Cuvello-Neto AL, Chocair PR. COVID-19 and cytomegalovirus co-infection: a challenging case of a critically ill patient with gastrointestinal symptoms. EJCRIM 2020;7: doi:10.12890/2020_001911.
\end{abstract}

Conflicts of Interests: The Authors declare that there are no competing interests.

This article is licensed under a Commons Attribution Non-Commercial 4.0 License

\section{ABSTRACT}

COVID-19 is a severe disease that has reached pandemic status. To the best of our knowledge, we describe the first case of COVID-19 and cytomegalovirus (CMV) co-infection in a critically ill patient. We discuss the challenge of establishing the diagnosis as well as the management of tissue-invasive gastrointestinal CMV infection (TI-GI CMV) simulating vascular involvement and intestinal obstruction in a critically ill patient.

\section{LEARNING POINTS}

- We describe a case of COVID-19 and cytomegalovirus (CMV) co-infection in a critically ill patient.

- Clinical symptoms simulated mesenteric vascular involvement and intestinal obstruction.

- The successful management of invasive CMV colitis in a patient with COVID-19 with atypical symptoms is described.

\section{KEYWORDS}

Colitis, COVID-19, cytomegalovirus, immunosuppression, intensive care unit

\section{INTRODUCTION}

Coronavirus disease 2019 (COVID-19) is caused by severe acute respiratory syndrome coronavirus 2 (SARS-CoV-2) and has rapidly spread worldwide. As of July 9, there were over 11 million confirmed cases globally and 545,481 deaths ${ }^{[1]}$. The first case in Brazil was reported on February 26 and 1,713,760 cases had been confirmed by early July ${ }^{[2]}$. In most cases, COVID-19 causes respiratory symptoms, such as dyspnoea, cough and coryza, but some patients may experience severe pneumonia, respiratory failure and organ failure ${ }^{[3]}$.

Critical illness is a risk factor for cytomegalovirus (CMV) reactivation due to immunosuppression ${ }^{[4]}$. Tissue-invasive gastrointestinal (TI-GI) $\mathrm{CMV}$ is most frequently reported in the colon and causes digestive symptoms such as diarrhoea, nausea and hyporexia ${ }^{[5]}$.

We describe the first case of COVID-19 and CMV co-infection in a critically ill patient, and discuss the challenge of establishing the diagnosis as well as the management of TI-GI CMV simulating mesenteric vascular involvement and intestinal obstruction in a critically ill patient with COVID-19.

\section{CASE DESCRIPTION}

A 62-year-old Caucasian male patient was admitted to the emergency room with a 1-week history of fever without cough or dyspnoea. Clinical examination revealed hypoxaemia $\left(\mathrm{SatO}_{2} 86 \%\right.$ ), while computed tomography (CT) of the thorax showed ground-glass opacities 
involving $50 \%$ of the lung parenchyma. A SARS-CoV-2 polymerase chain reaction (PCR) test was positive.

By day 7 of hospitalization, the patient had developed severe acute respiratory distress syndrome (ARDS), and was being managed with orotracheal intubation, vasoactive drugs, corticosteroids, prone position and prophylaxis for venous thromboembolism (VTE). Nevertheless, he remained hypoxaemic and was maintained on extracorporeal membrane oxygenation from the 10th to the 20th day of hospitalization. Then, he presented a hemothorax due to an expontaneous pulmonary bleeding managed by thoracotomy and a partial inferior lobectomy. He also developed acute renal failure requiring dialysis. However, despite his severe condition, over the next 3 weeks, the patient improved clinically while continuing with dialysis but without oxygen supplementation or haemodynamic instability. On day 30 of hospitalization, he presented with abdominal distention and pain. An abdominal CT scan revealed jejunal thickening with no evidence of mesenteric ischaemia (Fig. 1A,B). At that time, we opted for clinical surveillance and the patient's clinical symptoms improved.

On day 38 of hospitalization, the patient complained again of abdominal distention, pain and nausea/vomiting and showed haemodynamic instability, lactic acidosis, leucocytosis and D-dimer levels worsening to $20,622 \mathrm{ng} / \mathrm{ml}$ FEU. This clinical picture suggested a vascular abdominal syndrome. A CT scan of the abdomen revealed small bowel thickening and vascular engorgement, without arterial obstruction. The patient showed spontaneous clinical improvement during surveillance, although with recurrent diarrhoea. However, 1 week later, symptoms recurred and abdominal compartment syndrome and lower limb livedo developed. A CT scan of the abdomen evidenced marked colic distention suggestive of an obstructive abdominal syndrome. Decompressive colonoscopy was performed and revealed sparse colonic and terminal ileal mucosal ulcerations, which were biopsied (Fig. 1C). Histological findings showed ulcerated lesions with inflammatory infiltrate and evidence of virus-induced cytopathic effects (Fig. 1D). Immunohistochemistry was positive for CMV infection and negative for herpes virus infection (Fig. 1E). Serum PCR for CMV reached 308,000 IU/ml. Ganciclovir was then started and 72 hours later abdominal distension resolved, diarrhoea reduced, and laboratory test results improved, permitting food to be administered to the patient through a nasogastric tube. He completely recovered and was discharged from hospital.

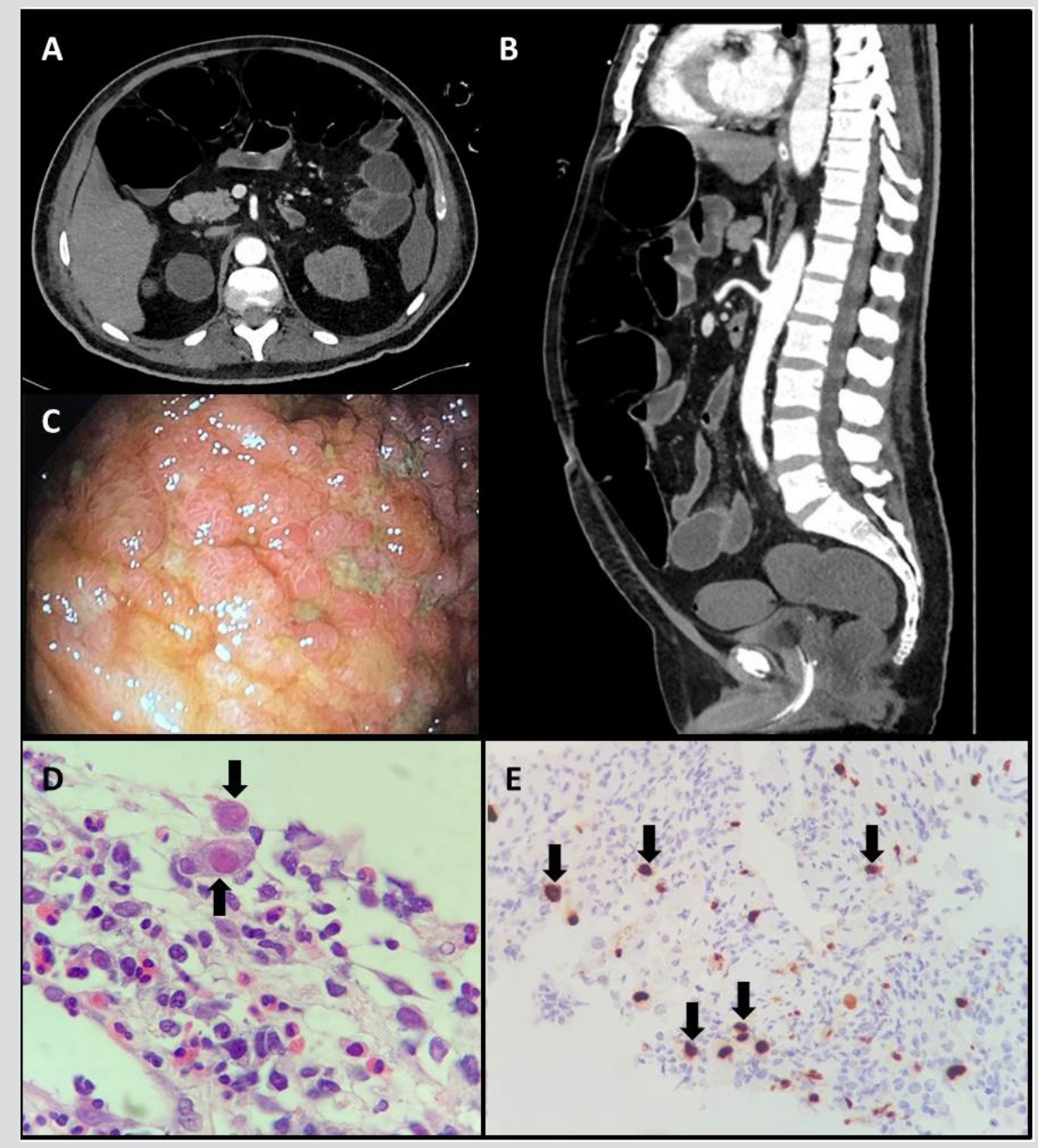

Figure 1. (A,B) Computed tomography scan of the abdomen revealed jejunal thickening with no evidence of mesenteric ischaemia. (C) Colonoscopy findings showed an extensive ulcerated area, measuring about $5.0 \mathrm{~cm}$, affecting $80 \%$ of the ileum circumference. (D) Biopsy of the ileum revealing extensive ulceration with fibrino-leucocytic exudate and endothelial cells with viral cytopathic changes (black arrows, $\times 400$ ). (E) Immunohistochemistry for cytomegalovirus showed positive staining in several cells (black arrows, $\times 100$ ) 


\section{DISCUSSION}

COVID-19 is a severe new disease and the management of critically ill patients is challenging since about $11 \%$ present with multiple organ failure ${ }^{[6]}$. An increasing number of studies have reported gastrointestinal involvement in COVID-19, with Cheung et al ${ }^{[7]}$ noting that $17.6 \%$ of their cohort in Hong Kong had gastrointestinal symptoms. In that report, the Gl symptoms started several days after diagnosis and patient hospitalization due to diarrhoea and abdominal distension. We discuss the challenges we faced managing our case below.

Challenge 1: Can gastrointestinal manifestations be attributed to COVID-19?

Faecal PCR testing for COVID-19 is as accurate as respiratory specimen PCR testing. In 53\% of faecal samples, PCR testing became positive and remained so in $82 \%$ of patients for several days after sputum excretion had become negative, as reported by Gu et al. ${ }^{[8]}$. Han et al. ${ }^{[9]}$ described a unique subgroup of patients with mild disease marked by the presence of digestive symptoms. Such patients are more likely to test positive for viral RNA in stool, to have a longer delay before viral clearance, and to experience delayed diagnosis. In this study of 206 patients with mild COVID-19, 48 patients had only digestive symptoms, 69 had simultaneous digestive and respiratory symptoms, and 89 had only respiratory symptoms. In 19.4\%, diarrhoea was the first symptom of the illness and lasted up to 14 consecutive days.

Fever was reported in $62.4 \%$ of patients with digestive symptoms. Patients with digestive symptoms had a longer duration between symptom onset and viral clearance. When there were respiratory and gastrointestinal symptoms, the virus was found in $73.3 \%$ of stool samples, while in the group with respiratory symptoms the virus was found in $14.3 \%$ of stool samples.

Virus RNA can be detected in stool samples in only $48.1 \%$ of COVID-19 patients ${ }^{[6]}$. Currently, this test is not available in Brazil. The late onset of gastrointestinal symptoms (30 days after disease onset) suggests COVID-19 is less likely the cause of the disease.

Challenge 2: Could abdominal symptoms be treated with surgery?

On day 38, the patient presented abdominal pain and distension, acidosis, high serum lactate levels and haemodynamic instability, since at that time he was not receiving any anticoagulation due to the haemothorax. As patients with COVID-19 may have a hypercoagulable state, we suspected a vascular ischaemic event or an obstruction.

The patient underwent abdominal CT angiography that revealed bowel wall thickening with no signs of mesenteric thrombosis or mechanic intestinal obstruction. Nor did it reveal abdominal free fluid or pneumatosis.

CT scanning reveals typical bowel abnormalities in COVID-19 patients. Bhayana et al. ${ }^{[10]}$ reported bowel wall abnormalities in $31 \%$ of patients. Bowel wall thickening was identified on $29 \%(n=12 / 42)$ of CT scans, and included colon/rectal thickening ( $n=7)$ and small bowel thickening $(n=5)$. In this study sample, small bowel thickening was exclusively seen in ICU patients. Bowel wall findings were common on CT and associated with ICU admission (OR 15.5, p=0.01).

These symptoms are non-specific and surgery in these patients, in addition to not being therapeutic, will cause morbidity and mortality due to fragility, consumption of the functional reserve, and immunosuppression. In critically ill patients, the main clinical indication for tomography was abdominal pain (33\%) and sepsis findings (29\% ${ }^{[10]}$.

Our patient showed partial improvement with clinical measures but presented a second time with a similar abdominal condition with worsening pain and distension. CT angiography again excluded acute vascular abdomen, intestinal obstruction, and other surgical pathologies.

Therefore, we considered a functional cause for the distention due to the lengthy hypomobility and multiple organ and metabolic dysfunction. We performed decompressive colonoscopy and in light of serum findings and positive immunohistochemistry for cytomegalovirus, we confirmed the diagnosis and started specific treatment with ganciclovir.

\section{Challenge 3: An association between SARS-CoV-2 and TI-GI CMV co-infection has not been previously described}

D'Ardes et al. ${ }^{[11]}$ reported the first case of co-infection with COVID-19 and CMV. Their 92-year-old patient had tested positive for SARSCoV-2 and was also investigated for CMV antibodies at presentation as he had high levels of IgG and IgM, suggesting an acute CMV infection. This patient had an unfavourable outcome after treatment with hydroxychloroquine and ritonavir/lopinavir and died 6 days after admission due to severe respiratory failure.

Reactivation of CMV may occur at any time, but is most common in the setting of immunosuppression. Osawa et al. described a patient in a critical condition, which is a known risk factor for CMV reactivation ${ }^{[12]}$. The patient had also received glucocorticoid for ARDS and refractory shock. The use of glucocorticoid is associated with an increased risk of CMV colitis in immunocompetent hosts ${ }^{[5]}$.

Recently, in this regard, Moss ${ }^{[13]}$ suggested that any such association might be reflected either by the extent of SARS-CoV-2 viral replication or by the quality of the subsequent immune response. A secondary influence exerted by the acute inflammation leading to enhanced CMV reactivation must also be considered. Further scientific investigation is necessary to establish this relationship and its clinical significance. 
We report successful management of invasive CMV colitis in a patient with COVID-19 with atypical symptoms. To our knowledge, this is the first case described with this co-infection. At the same time, we draw attention to this connection as we are dealing with an increasing number of critically ill patients treated with glucocorticoids, anti-IL-6 and other immunobiological therapies. Such treatments and maybe COVID-19 infection itself may have a direct effect on CMV infection/reactivation.

\section{REFERENCES}

1. WHO. Coronavirus disease 2019 (COVID-19) Situation Report - 94. https://www.who.int/docs/default-source/coronaviruse/situation-reports/20200423-sitrep-94-covid-19. pdf?sfvrsn=b8304bf0_4 (accessed 1 Aug 2020).

2. Health Ministry, Brazilian Government. COVID19 Painel Coronavirus. https://covid.saude.gov.br (accessed 9 July 2020).

3. Huang C, Wang Y, Li X, Ren L, Zhao J, Hu Y, et al. Clinical features of patients infected with 2019 novel coronavirus in Wuhan, China. Lancet 2020;395(10223):497-506.

4. Patra S, Samal SC, Chacko A, Mathan VI, Mathan MM. Cytomegalovirus infection of the human gastrointestinal tract. J Gastroenterol Hepatol 1999;14(10):973-976.

5. Fakhreddine AY, Frenette CT, Konijeti GG. A practical review of cytomegalovirus in gastroenterology and hepatology. Gastroenterol Res Pract 2019;2019:6156581.

6. Chen N, Zhou M, Dong X, Qu J, Gong F, Han Y, et al. Epidemiological and clinical characteristics of 99 cases of 2019 novel coronavirus pneumonia in Wuhan, China: a descriptive study. Lancet 2020;395(10223):507-513.

7. Cheung KS, Hung IF, Chan PP, Lung KC, Tso E, Liu R, et al. Gastrointestinal manifestations of SARS-CoV-2 infection and virus load in fecal samples from a Hong Kong cohort: systematic review and meta-analysis. Gastroenterology 2020;159(1):85-95.

8. Gu J, Han B, Wang J. COVID-19: gastrointestinal manifestations and potential fecal-oral transmission. Gastroenterology 2020;158(6):1518-1519.

9. Han C, Duan C, Zhang S. Digestive symptoms in COVID-19 patients with mild disease severity: clinical presentation, stool viral RNA testing and outcomes. Am J Gastroenterol 2020;115(6):916-923.

10. Bhayana R, Som A, Li ML. Abdominal imaging findings in COVID-19: preliminary observations. Radiology 2020 May 11. doi: 10.1148/radiol. 201908 [Epub ahead of print].

11. D'Ardes D, Boccatonda A, Schiavone C, Santilli F, Guagnano MT, Bucci M, et al. A case of coinfection with SARS-CoV-2 and cytomegalovirus in the era of COVID-19. Eur J Case Rep Intern Med 2020;7(5):001652.

12. Osawa R, Singh N. Cytomegalovirus infection in critically ill patients: a systematic review. Crit Care 2009;13(3):R68.

13. Moss $P$. "The ancient and the new": is there an interaction between cytomegalovirus and SARS-CoV-2 infection? Immun Ageing 2020;17:14. 